Check for updates

Cite this: RSC Adv., 2019, 9, 466

\title{
Electrochemical estimation of active site density on a metal-free carbon-based catalyst $\uparrow$
}

\author{
Arup Chakraborty, ${ }^{a}$ Bapi Bera, ${ }^{a}$ Divya Priyadarshani, ${ }^{\text {b }}$ Pradipkumar Leuaa, ${ }^{a}$ \\ Debittree Choudhury ${ }^{a}$ and Manoj Neergat (D)*a
}

Nitrogen-doped carbon is synthesized by the heat-treatment of carbon in an ammoniacal atmosphere at different temperatures. The active site density and electrochemically active surface area (ESA) of carbon and nitrogen-doped carbon catalysts are estimated from the charge due to oxidation of the adsorbed anthraquinone-2-sulfonate (AQS) probe molecule. In the potential window of interest and over a range of concentrations, there is no unwanted side reaction or polymerization of the probe molecule that interferes with the electrochemical estimation of active site density. Most importantly, the adsorbed AQS can easily be removed from the electrode surface by potential cycling. The ORR activity and active site density of the catalysts derived from AQS-adsorption have similar trends. The active site density and turnover frequency towards ORR estimated using the AQS-adsorption method are in line with those reported in the literature by other methods. On the other hand, the results show that the wetted surface area estimated from the double layer capacitance does not always correlate with catalytic activity.

Received 27th October 2018 Accepted 18th December 2018

DOI: 10.1039/c8ra08906j

rsc.li/rsc-advances and Mössbauer spectroscopy were also used to identify the possible ORR active centers in an Fe/N-C catalyst. ${ }^{\mathbf{1 0 - 1 3}}$ Recently, low temperature pulse CO chemisorption was applied to estimate the active site density of NPMCs, assuming the metal as the only ORR active center. ${ }^{\mathbf{1 4}}$ It was reported that, even at low temperatures $(193 \pm 5 \mathrm{~K})$, CO does not adsorb on the nitrogen center of the catalyst. In another report, $\mathrm{NO}_{(\mathrm{g})}$ was used as the probe molecule to detect the iron center in $\mathrm{Fe} / \mathrm{N}-\mathrm{C}$ catalyst. ${ }^{13}$ Thus, development of an electrochemical method to estimate the active site density of metal-free carbon and nitrogen-doped carbon is paramount.

In our previous work, a catechol molecule was used as a probe to estimate the active site density. ${ }^{15}$ The experiment was conducted based on the method proposed by Kanazawa et al. and was performed with minor modification. ${ }^{16}$ In this method, the in situ generated oxidized products of catechol bind to the active sites on the catalyst surface. This method yields reasonable estimate of the active site density at low concentration of catechol in the limited potential window for optimized adsorption duration. The base of the voltammogram of catechol-adsorbed electrode overlaps with the background voltammogram only at low concentrations, i.e., the capacitive contribution to the voltammograms of catechol-adsorbed electrode matches with the capacitance of the respective bare electrode. $^{15}$

At higher concentrations of catechol, unwanted electrochemical reactions occur and the polymerized catechol adsorbs on the electrode surface as proposed by others. ${ }^{17-19}$ This leads to an overestimation of the catechol adsorption charge and the active site density. Furthermore, the base of the voltammogram
${ }^{a}$ Department of Energy Science and Engineering, Indian Institute of Technology Bombay (IITB), Powai, Mumbai-400076, India.E-mail: nmanoj@iitb.ac.in; Fax: + 91222576 4890; Tel: + 912225767893

${ }^{b}$ Centre for Research in Nanotechnology \& Science, Indian Institute of Technology Bombay (IITB), Powai, Mumbai-400076, India

$\dagger$ Electronic supplementary information (ESI) available. See DOI: 10.1039/c8ra08906j 
of catechol-adsorbed electrode recorded after cycling in catechol solution of higher concentration does not overlap with the background voltammogram in the limited or wider potential window, i.e., the capacitive contribution in the voltammograms of catechol-adsorbed electrode does not match with the bare electrode. In such conditions, it is difficult to estimate the active site density of the catalyst.

On the other hand, while using the anthraquinone-2sulfonate (AQS) molecule, a single saturated peak of the adsorbed AQS voltammogram is obtained at any concentration. Irrespective of the AQS concentration, the base of the adsorption voltammogram matches with the background voltammogram recorded in AQS-free supporting electrolyte.

Therefore, AQS is used as a probe molecule to estimate the active site density. This method provides reproducible results over a wide range of AQS concentrations and potentials. Moreover, it can be easily removed from the electrode surface, as compared to that of catechol.

The ORR voltammograms are recorded on carbon, nitrogendoped carbon and the $\mathrm{HNO}_{3}$ treated catalysts in argon-saturated $0.1 \mathrm{M} \mathrm{KOH}$ media. It is observed that the ORR activity and the active site density estimated from the AQS-adsorption method have similar trends. Furthermore, the wetted surface area of the catalysts is calculated from the double layer capacitance estimated using electrochemical impedance spectroscopy (EIS). It is shown that the catalytic activity may not always correlate with the wetted surface area.

\section{Experimental details}

\subsection{Materials}

Anthraquinone-2-sulfonic acid sodium salt monohydrate (AQS; $\mathrm{C}_{14} \mathrm{H}_{7} \mathrm{NaO}_{5} \mathrm{~S} \cdot \mathrm{H}_{2} \mathrm{O}$ ), 1,2-dihydroxybenzene (catechol; 1,2$\mathrm{C}_{6} \mathrm{H}_{4}(\mathrm{OH})$ ) and Nafion ${ }^{\circledR}$ suspension (5 wt\% solution in lower aliphatic alcohols $/ \mathrm{H}_{2} \mathrm{O}$ mix) were purchased from Sigma Aldrich. 2-Propanol $\left(\left(\mathrm{CH}_{3}\right)_{2} \mathrm{CHOH}\right)$, potassium dihydrogen phosphate $\left(\mathrm{KH}_{2} \mathrm{PO}_{4}\right)$, dipotassium hydrogen phosphate $\left(\mathrm{K}_{2} \mathrm{HPO}_{4}\right)$, potassium chloride $(\mathrm{KCl})$, nitric acid $\left(\mathrm{HNO}_{3}\right)$ and ammonia $\left(\mathrm{NH}_{3}\right)$ were purchased from Merck. All the chemicals were used without further purification. De-ionized (DI) water was obtained from Direct-Q Millipore de-ionizer.

\subsection{Synthesis of $\mathbf{N}$-doped carbon (N/C)}

$\mathrm{N}$-doped carbon was synthesized by the heat-treatment of $100 \mathrm{mg}$ carbon black (Vulcan XC-72) with nitrogen precursor at appropriate temperature. ${ }^{15,20,21}$ Prior to the heat-treatment, the carbon sample, taken in a dry quartz boat and kept in a quartz tubular furnace, was purged with argon for $15 \mathrm{~min}$. After purging out the air inside the furnace, the carbon sample was heat-treated in an ammoniacal argon (argon gas purged through $30 \%$ ammonia solution) atmosphere for nitrogendoping. After the heat-treatment at the desired temperature for three hours, the furnace was allowed to cool to room temperature. The collected sample was ground well in a mortar with a pestle and was preserved for further characterization. The samples heat-treated at 1000, 900, 800 and $700{ }^{\circ} \mathrm{C}$ are labeled as N/C 1000, N/C 900, N/C 800, N/C 700, respectively, in this manuscript.

\section{3. $\mathrm{HNO}_{3}$ treatment of carbon}

Carbon black (Vulcan XC-72) ( $100 \mathrm{mg}$ ) was dispersed in $50 \mathrm{~mL}$ $\mathrm{HNO}_{3}(69 \%)$ solution and was stirred overnight at $80{ }^{\circ} \mathrm{C}$. The dispersion was centrifuged at $10000 \mathrm{rpm}$ rotation to get the black residue. The residue was washed repeatedly with DI water by centrifugation at $10000 \mathrm{rpm}$ to get the neutralized supernatant. The cleaned residue was kept in a hot-air oven $\left(\sim 80^{\circ} \mathrm{C}\right)$ to dry it. The sample was collected and ground in a mortar pestle to get the uniform powder. The powder was used for further characterization and labeled as $\mathrm{HNO}_{3}$ treated carbon. $^{22,23}$

\subsection{Electrochemical characterization}

Cyclic voltammograms were recorded with a Bi-potentiostat ("Wavedriver 20" from Pine Instrument USA) in a threeelectrode cell configuration. Bio Logic SP300 (from France) was used to record the impedance spectra in a three-electrode configuration. AC amplitude of $5 \mathrm{mV}$ rms was used to record the impedance data in the frequency range of $10 \mathrm{kHz}$ to $50 \mathrm{mHz}$ and the same were analyzed using the complex non-linear least square (CNLS) method with "ZSimpWin" software. A double junction $\mathrm{Ag} / \mathrm{AgCl}$ (saturated with $\mathrm{KCl}$ ) was used as a reference electrode and high surface area platinum coil as a counter electrode. A polished GCE (geometric surface area $0.196 \mathrm{~cm}_{\mathrm{g}}{ }^{2}$ ) and catalyst deposited GCE were used as the working electrodes. Prior to the experiments, GCE was polished using alumina MasterPolish suspension from Buehler and was cleaned with DI water by one-minute ultrasonication. Catalyst ink was prepared by sonicating a mixture of $5 \mathrm{mg}$ catalyst with $5 \mathrm{~mL}$ DI water, $10 \mathrm{~mL}$ iso-propanol and $10 \mu \mathrm{L}$ Nafion ${ }^{\circledR}$ solution for $30 \mathrm{~min}^{24}$ The well-dispersed ink was drop-cast on a polished GCE to get a loading of $\sim 136 \mu \mathrm{g} \mathrm{cm}_{\mathrm{g}}{ }^{-2}$; the electrode was dried at room temperature for $2 \mathrm{~h}$. The voltammograms were recorded in the desired electrolyte at a scan rate of $20 \mathrm{mV} \mathrm{s}^{-1}$. All the potentials are reported with respect to standard $\mathrm{Ag} / \mathrm{AgCl}$ (saturated $\mathrm{KCl}$ ) electrode in this manuscript. The ORR activity of the catalyst was recorded in oxygen-saturated $0.1 \mathrm{M} \mathrm{KOH}$ solution.

2.4.1 Adsorption of anathraquinone-2-sulphonate and catechol. Prior to recording the voltammograms of AQSadsorbed electrode, the electrode was activated following the procedure as mentioned in the Section 2.3.2 and then the background voltammograms (20 cycles) were recorded in argonsaturated 0.1 M PBS ( $\mathrm{pH} 7$ ) solution at $20 \mathrm{mV} \mathrm{s}^{-1}$ scan rate in the potential window of -0.65 to $0.55 \mathrm{~V}$. The electrode was then cycled in the same potential window in argon-saturated AQS solution (prepared either in 0.1 M PBS ( $\mathrm{pH} 7$ ) or in DI water) to adsorb the AQS on the surface. The electrode was cleaned in $0.1 \mathrm{M}$ PBS ( $\mathrm{pH} \mathrm{7}$ ) for $20 \mathrm{~min}$. Under rotation $(1600 \mathrm{rpm})$ to remove the excess amount of AQS physically trapped in the thin layer of the electrode. Finally, after removal of unbound porevolume trapped AQS, the voltammogram of AQS-adsorbed electrode was recorded in fresh (AQS-free) argon-saturated $0.1 \mathrm{M}$ PBS (pH 7) solution. AQS is soluble in 0.1 M PBS (pH 7) 
only upto $\sim 1 \mathrm{mM}$, whereas in DI water, it is soluble upto $20 \mathrm{mM}$. Thus, the electrode is cycled to adsorb AQS on the surface at different concentrations of AQS upto $20 \mathrm{mM}$.

The catechol adsorption was performed by the method reported earlier by our group. ${ }^{15}$

2.4.2 KOH treatment. The catalysts were cycled in argonsaturated $0.1 \mathrm{M} \mathrm{KOH}$ solution in the potential window of -1.0 to $0 \mathrm{~V} v s$. $\mathrm{Ag} / \mathrm{AgCl}$ at a scan rate $20 \mathrm{mV} \mathrm{s}^{-1}$ for 20 cycles either to activate or to electrochemically remove the adsorbed molecules. The $\mathrm{KOH}$-treated samples were then cleaned with $0.1 \mathrm{M}$ PBS (pH 7) at $1600 \mathrm{rpm}$. The cleaned samples were used for recording the adsorbed AQS or catechol voltammogram, as mentioned above.

2.4.3 Oxygen reduction reaction (ORR). The ORR voltammogram was recorded in $0.1 \mathrm{M} \mathrm{KOH}$ solution in an $\mathrm{RDE}$ configuration at $1600 \mathrm{rpm}$. Prior to the experiment, the solution was saturated with oxygen by purging for $20 \mathrm{~min}$. The voltammogram was recorded in the potential window of -1.0 to $0.0 \mathrm{~V}$ at a scan rate of $20 \mathrm{mV} \mathrm{s}^{-1}$.

\subsection{Physical characterization}

X-ray photoelectron spectroscopy (XPS) of the catalyst samples was recorded with Kratos Analytical, UK spectrometer using Al $\mathrm{K} \alpha$ radiation. The spectrum was fitted with XPS peak fit 4.1 software. The BET surface area of the porous catalysts was measured using the Smart instrument BET surface analyzer (Smart Sorb 92/93).

\section{Results and discussion}

\subsection{Voltammograms of catechol-adsorbed and AQS- adsorbed N/C 900}

Fig. 1 shows the voltammograms of catechol-adsorbed N/C 900 catalyst recorded in catechol-free argon-saturated $0.1 \mathrm{M}$ PBS $(\mathrm{pH} 7)$ along with the respective background voltammogram in the potential window of -0.65 to $0.55 \mathrm{~V}$. The catalyst is cycled in catechol solutions of different concentrations and then the voltammogram of the catechol-adsorbed electrode is recorded in argon-saturated 0.1 M PBS ( $\mathrm{pH} 7$ ). In the previous work, the voltammograms of catechol-adsorbed electrode were recorded in the potential window of -0.15 to $0.55 \mathrm{~V}$, and $1 \mathrm{mM}$ catechol was used to adsorb catechol (shown in the inset of Fig. 1). ${ }^{15}$ The origin of the two peaks observed in the voltammogram of catechol-adsorbed electrode was discussed in detail in a previous manuscript. ${ }^{15}$ The experiment was performed on the basis of the method proposed by Kanazawa et al., but with minor modification. ${ }^{16}$ During the potential cycling of the catalyst in the catechol solution, the in situ generated oxidized products of catechol adsorb on the active sites of the catalyst. Under the experimental conditions, it was observed that the base of adsorption voltammogram matches with the background voltammogram and it was assumed that the active sites on the catalyst surface binds with the catechol molecule.

Whereas, as shown in Fig. 1, at the extended potential window of -0.65 to $0.55 \mathrm{~V}$, with $1 \mathrm{mM}$ and higher concentration of catechol solution, the double layer region of the voltammogram for adsorbed-catechol electrode expands and the

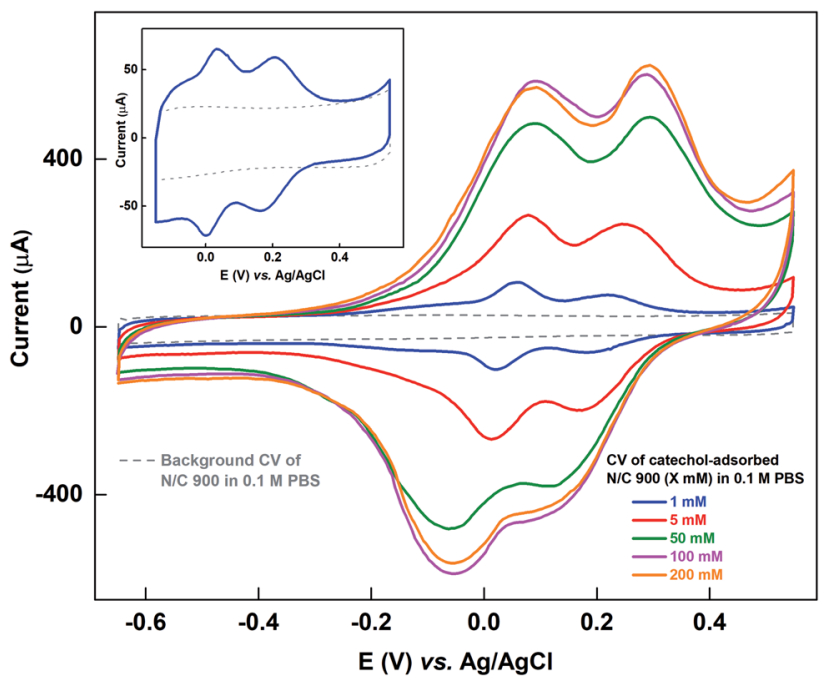

Fig. 1 Voltammograms of background (gray) and catechol-adsorbed N/C 900 after potential cycling in catechol solutions of different concentrations (shown in different colors) in argon-saturated $0.1 \mathrm{M}$ PBS ( $\mathrm{pH} \mathrm{7)} \mathrm{at} 20 \mathrm{mV} \mathrm{s}^{-1}$ scan rate. Inset shows the voltammogram of catechol-adsorbed N/C 900 recorded in limited potential window $(-0.15$ to $0.55 \mathrm{~V})$ after potential cycling in $1 \mathrm{mM}$ catechol along with the background voltammogram (gray).

background correction is erroneous, as the straight-line correction method is used to estimate the adsorbed-catechol charge.

Table $\mathrm{S} 1 \uparrow$ shows the charge due to adsorbed-catechol on N/C 900 catalyst at different concentrations. The charge for the catechol adsorption is estimated after the straight-line correction from the voltammogram of catechol-adsorbed catalysts as shown in Fig. S1.† It increases with the catechol concentration and almost saturates at $\sim 6.8 \mathrm{mC}$ with $100 \mathrm{mM}$ and $200 \mathrm{mM}$ catechol concentrations

It is believed that, in case of the catechol-adsorption method, the oxidized products generated with potential cycling adsorb on the catalyst surface. ${ }^{16}$ Thus, instead of catechol or any other reduced probes, if an oxidized compound, which adsorbs on carbon can be supplied as the probe molecule, it can easily be attached on the surface at lower concentration of redox species. Also, the AQS-adsorption peak appears at such a potential, where no side reaction like hydrogen evolution can take place. Thus, AQS has been chosen as an adsorbate.

Similar kinds of experiments, as that with catechol molecule, are performed at different concentrations to get the saturated surface-coverage with the probe molecule. Fig. 2(a) shows the voltammogram of N/C 900 catalyst recorded in the potential range of -0.65 to $0.55 \mathrm{~V}$ in $0.1 \mathrm{mM}$ AQS dissolved in $0.1 \mathrm{M}$ PBS $(\mathrm{pH} 7)$ solution (red). It takes $\sim 40$ potential cycles to get the electrode surface saturated with AQS (overlapping voltammograms). Whereas, while using the $1 \mathrm{mM}$ AQS, the electrode surface gets saturated by 4-5 potential cycles as shown in the voltammogram of Fig. 2(b) (red). As the experiments are performed on completely wetted surface of the catalyst, the increment of the current with potential cycles is due to the accumulation of the AQS molecule on the catalyst surface. 


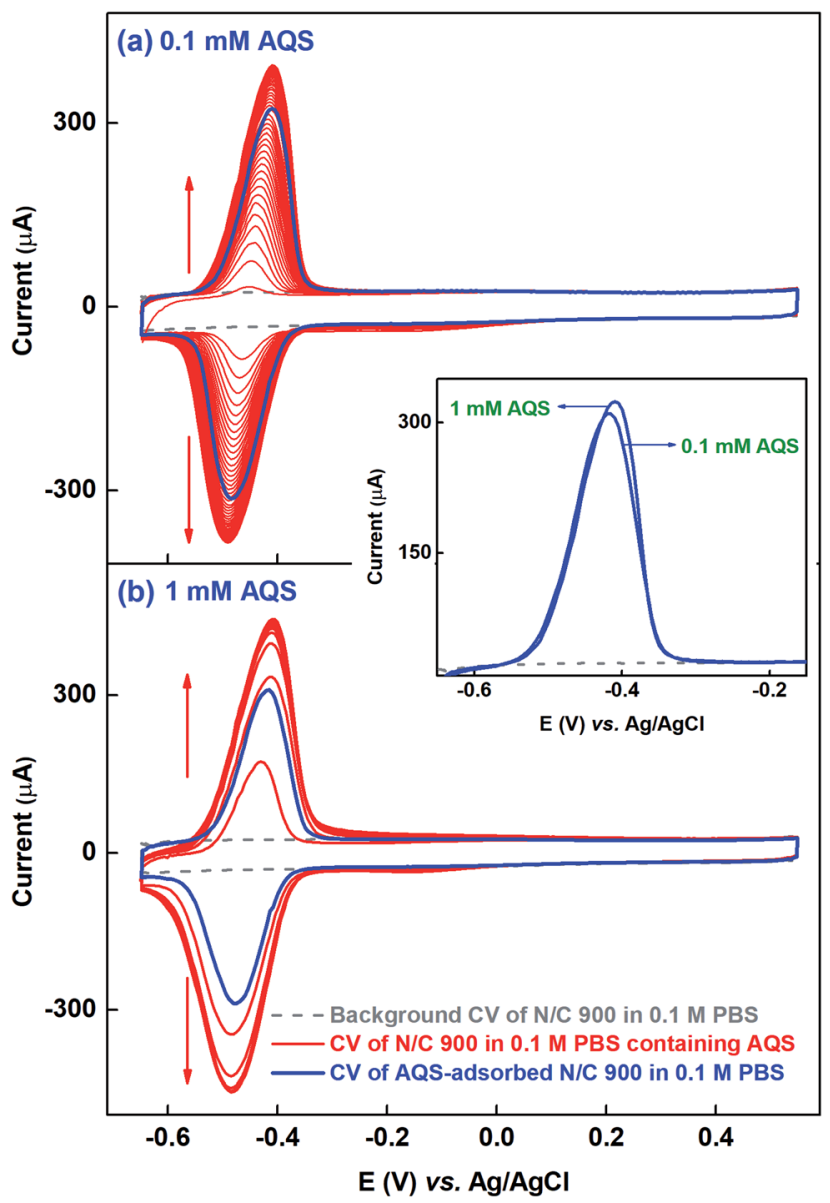

Fig. 2 Voltammograms of N/C 900 recorded in argon-saturated 0.1 M PBS ( $\mathrm{pH} 7)$ at $20 \mathrm{mV} \mathrm{s}^{-1}$ scan rate at different conditions. Background CV (gray); cycling CV in $0.1 \mathrm{mM}$ (a), and $1 \mathrm{mM}$ (b) AQS (red); and CV of AQS-adsorbed electrode after potential cycling in $0.1 \mathrm{mM}$ (a) and $1 \mathrm{mM}$ (b) AQS (blue). Inset shows the overlapping voltammograms (oxidation peak from the forward cycle) of the AQS-adsorbed electrode after potential cycling in $0.1 \mathrm{mM}$ and $1 \mathrm{mM}$ AQS.

After potential cycling in bulk AQS solution, the electrode is washed by rotating at $1600 \mathrm{rpm}$ for an optimized time of $20 \mathrm{~min}$ in $0.1 \mathrm{M}$ PBS ( $\mathrm{pH} 7$ ) to remove the pore volume trapped-AQS, i.e., unbound or weekly bound AQS from the catalyst surface. Subsequently, the electrode is potential cycled in $0.1 \mathrm{M} \mathrm{PBS} \mathrm{(pH}$ 7) to record the voltammogram of AQS-adsorbed N/C 900, shown in Fig. 2 (blue voltammogram). The integrated charge from the voltammogram of AQS-adsorbed N/C 900 (blue) is lower than that of the voltammogram of N/C 900 (red) recorded in bulk AQS solution. The decrease in charge justifies the requirement of washing the catalyst surface in AQS-free supporting electrolyte prior to recording the voltammogram of AQSadsorbed electrode in $0.1 \mathrm{M}$ PBS ( $\mathrm{pH} 7$ ).

The redox peak of voltammograms is centered at $\sim-0.44 \mathrm{~V}$ and the possible redox reaction is a two-electron process, given in Scheme $1 .^{25}$

For comparison, the oxidation peak from the voltammograms of AQS-adsorbed N/C 900 after potential cycling in $0.1 \mathrm{mM}$ and $1 \mathrm{mM}$ AQS are shown in the inset to Fig. 2. The base of the voltammograms of AQS-adsorbed electrode matches with<smiles></smiles>

Scheme 1

the background voltammogram at the lower and the higher potentials. Thus, it is possible to correct for the background charge properly and to calculate the charge due to surfaceadsorbed AQS correctly. The comparable charge $(\sim 1.40 \mathrm{mC})$ from both the background corrected voltammograms of AQSadsorbed N/C 900 suggests that active sites of the catalyst are saturated with AQS and it is assumed that each active site on the catalyst surface adsorbs one AQS molecule; further evidence towards the aforementioned is discussed below.

As the solubility of AQS in $0.1 \mathrm{M} \mathrm{PBS}(\mathrm{pH} 7)$ is poor $(\sim 1 \mathrm{mM})$ at room temperature, and it is $\sim 20 \mathrm{mM}$ in DI water, the N/C 900 is allowed to adsorb AQS from its aqueous solution of different concentrations. Fig. 3 shows the voltammograms of the AQSadsorbed N/C 900 recorded in AQS-free 0.1 M PBS after potential cycling in three different concentrations of aqueous AQS solutions $(1,10$, and $20 \mathrm{mM})$. Inset to Fig. 3 shows the voltammograms of N/C 900 electrode during the potential cycling in water containing AQS solution. It is observed that the oxidation and reduction current increase with the concentration of AQS during the potential cycling. Whereas, the oxidation charge of the adsorbed species does not change as shown in the respective voltammograms of AQS-adsorbed electrode. It suggests that the active sites on the catalyst surface are

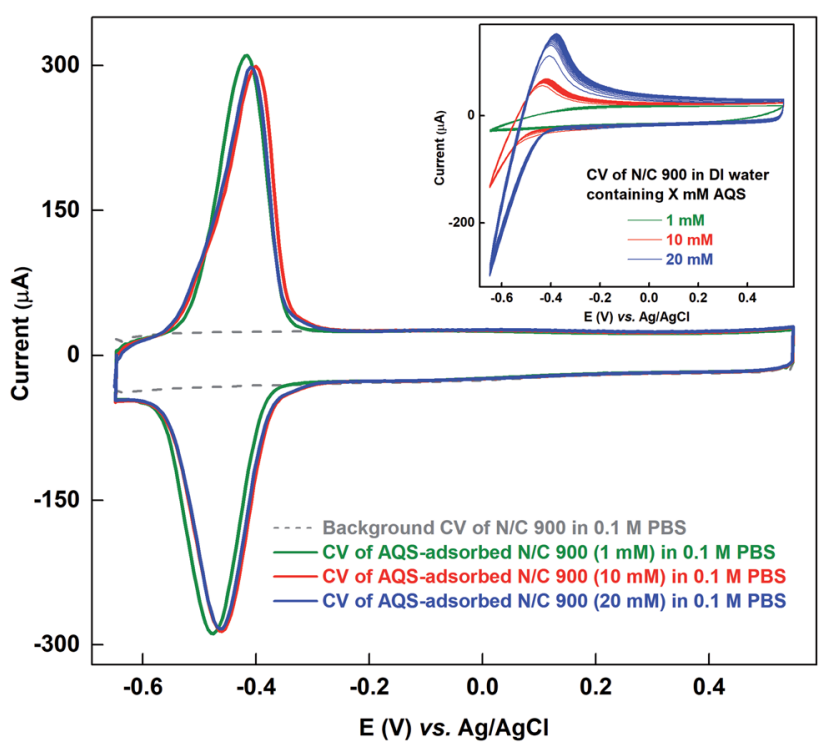

Fig. 3 Voltammogram of AQS-adsorbed N/C 900 recorded in argonsaturated AQS-free $0.1 \mathrm{M} \mathrm{PBS}\left(\mathrm{pH}\right.$ 7) at $20 \mathrm{mV} \mathrm{s}^{-1}$ scan rate after cycling in AQS-containing DI water. Gray dotted line represents the background voltammogram of N/C 900 recorded in AQS-free argonsaturated $0.1 \mathrm{M}$ PBS ( $\mathrm{pH}$ 7). Inset shows the voltammograms of N/C 900 for the potential cycling in aqueous AQS solutions of different concentrations. 
saturated with AQS. Moreover, the charge due to oxidation of adsorbed-AQS obtained after potential cycling in aqueous solution of AQS is comparable with that of the catalyst cycled in $0.1 \mathrm{M}$ PBS (pH 7) ( 1.4 mC). It further confirms that the AQS adsorbed on the catalyst surface is of same quantity (inset of Fig. 2 and main figure of Fig. 3). Thus, it can be used to estimate the active site density of the catalyst convincingly.

Furthermore, the problem related to the catechol-adsorption method is investigated by replacing the surface-adsorbed AQS with catechol molecule. This discussion is given in the ESI. $\dagger$

\subsection{Recovery of the active sites}

The active sites on the surface of the AQS-adsorbed electrode can be recovered by cycling the electrode in argon-saturated $0.1 \mathrm{M} \mathrm{KOH}$ in the potential range of -1.0 to $0.0 \mathrm{~V}$. Fig. 4 shows the cyclic voltammograms of the AQS-adsorbed N/C 900 in argon-saturated $0.1 \mathrm{M} \mathrm{KOH}$ media. After the initial 4-5 cycles, no adsorption peak is observed. The cleaned catalyst is again cycled in 0.1 M PBS ( $\mathrm{pH} 7$ ) to check the effectiveness of electrochemical cleaning.

Fig. 5 shows the comparison of the voltammograms of AQSadsorbed N/C 900 and after recovery of the active sites by $\mathrm{KOH}$ cleaning with that of the fresh N/C 900. AQS binds with the surface during the adsorption. It is necessary to ensure that the adsorbed molecule can be removed, after estimating the active sites. For this, the $\mathrm{KOH}$ cleaning method is used. On a cleaned electrode, AQS is adsorbed again by potential cycling (as in Fig. 2). The electrode is washed to remove pore-volume trapped AQS for $20 \mathrm{~min}$. The voltammogram is subsequently recorded in AQS-free 0.1 M PBS (pH 7) supporting electrolyte. Comparable adsorption charge $(\sim 1.4 \mathrm{mC})$ is obtained for both voltammograms in Fig. 5. The overlapping background voltammograms of N/C 900 and that of the recovered surface as shown in the inset of Fig. 5 supports the effectiveness of electrochemical cleaning method.

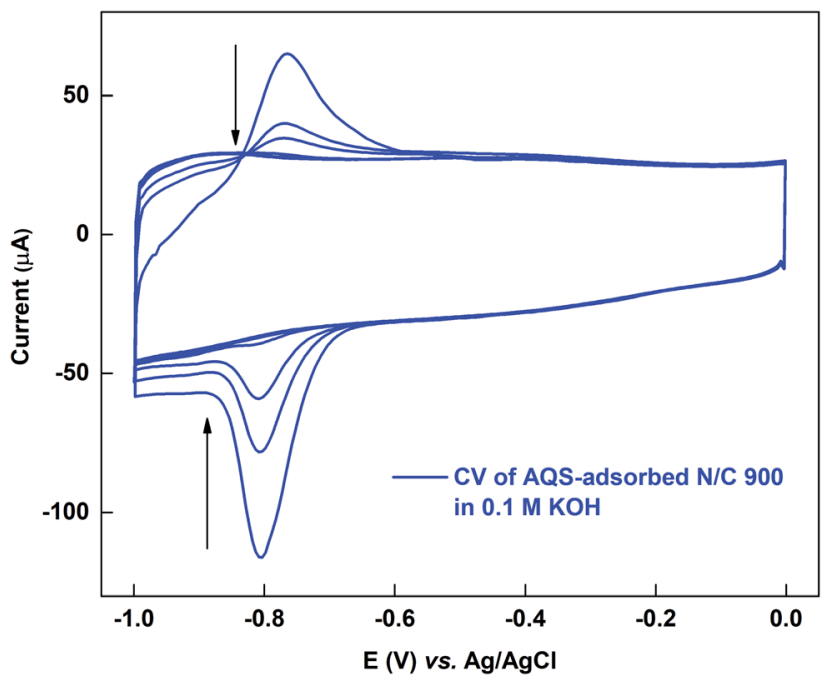

Fig. 4 Voltammogram of AQS-adsorbed N/C 900 potential cycled in argon-saturated $0.1 \mathrm{M} \mathrm{KOH}\left(\mathrm{pH} \mathrm{13)}\right.$ at $20 \mathrm{mV} \mathrm{s}^{-1}$ scan rate.

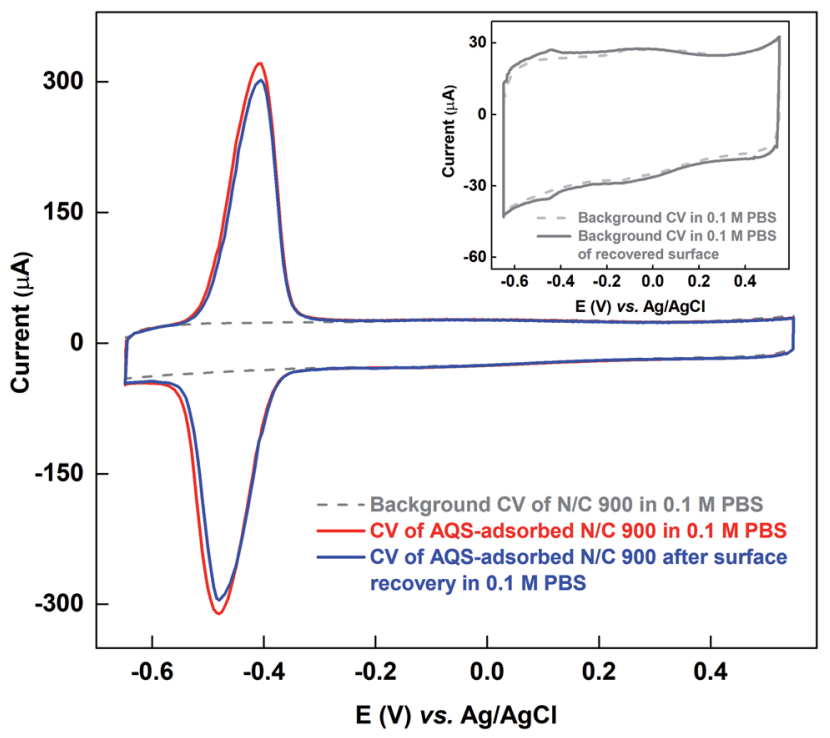

Fig. 5 Voltammograms of N/C 900 catalyst recorded in argon-saturated $0.1 \mathrm{M}$ PBS ( $\mathrm{pH} 7)$ at $20 \mathrm{mV} \mathrm{s}^{-1}$ scan rate at different conditions; background (gray), AQS-adsorbed electrode (red), and with the recovered surface (blue). The inset represents the comparison of the background voltammograms of the initial and the recovered surfaces of N/C 900 after electrochemical cleaning in $\mathrm{KOH}$ media.

\subsection{Estimation of the active site density, electrochemically active surface area (ESA) and wetted surface area of catalysts: correlation with ORR activity}

The voltammograms of AQS-adsorbed electrode is used to estimate the active site density and ESA of the catalysts, and the estimated active sites are correlated with their ORR activity. The wetted surface area is estimated from the double layer capacitance measured using the EIS in AQS-free argon-saturated $0.1 \mathrm{M}$ PBS ( $\mathrm{pH} \mathrm{7),} \mathrm{where} \mathrm{the} \mathrm{electrochemical} \mathrm{surface} \mathrm{behaves} \mathrm{like}$ a capacitor, and faradaic contribution is negligible. Nitrogendoped carbon catalysts are used initially for these estimations, and thereafter, the data with $\mathrm{HNO}_{3}$ treated catalysts are presented. At the end, the difference between the ESA and wetted surface area are discussed.

\subsubsection{Discussion on nitrogen-doped carbon catalyst}

3.3.1.1 Voltammograms of AQS-adsorbed nitrogen-doped carbon. The nitrogen-doped carbon catalysts are prepared by the heat-treatment of the carbon in ammonia atmosphere at various temperatures. The surface nitrogen content (at\%) of the catalysts measured from the XPS is provided in Table S3. $\dagger$ It confirms that the carbon surface is doped with nitrogen. It is observed that the surface nitrogen content is highest $(\sim 1.06$ at\%) for N/C 900 among all the nitrogen-doped catalysts. During the heat-treatment, the BET surface area and surface oxygen content (at\%) of the nitrogen-doped carbon changes as shown in Table $\mathrm{S} 3 \dagger$ and it is in the order N/C $1000>\mathrm{N} / \mathrm{C} 900>\mathrm{N} / \mathrm{C} 800$ $>\mathrm{N} / \mathrm{C} 700>\mathrm{C}^{15}$ The increment of BET surface area suggests that the surface area increases with the heat-treatment temperature.

Fig. 6 shows the voltammograms of AQS-adsorbed nitrogendoped carbon recorded in argon-saturated AQS-free 0.1 M PBS $(\mathrm{pH} 7)$ with their respective background voltammograms. To 


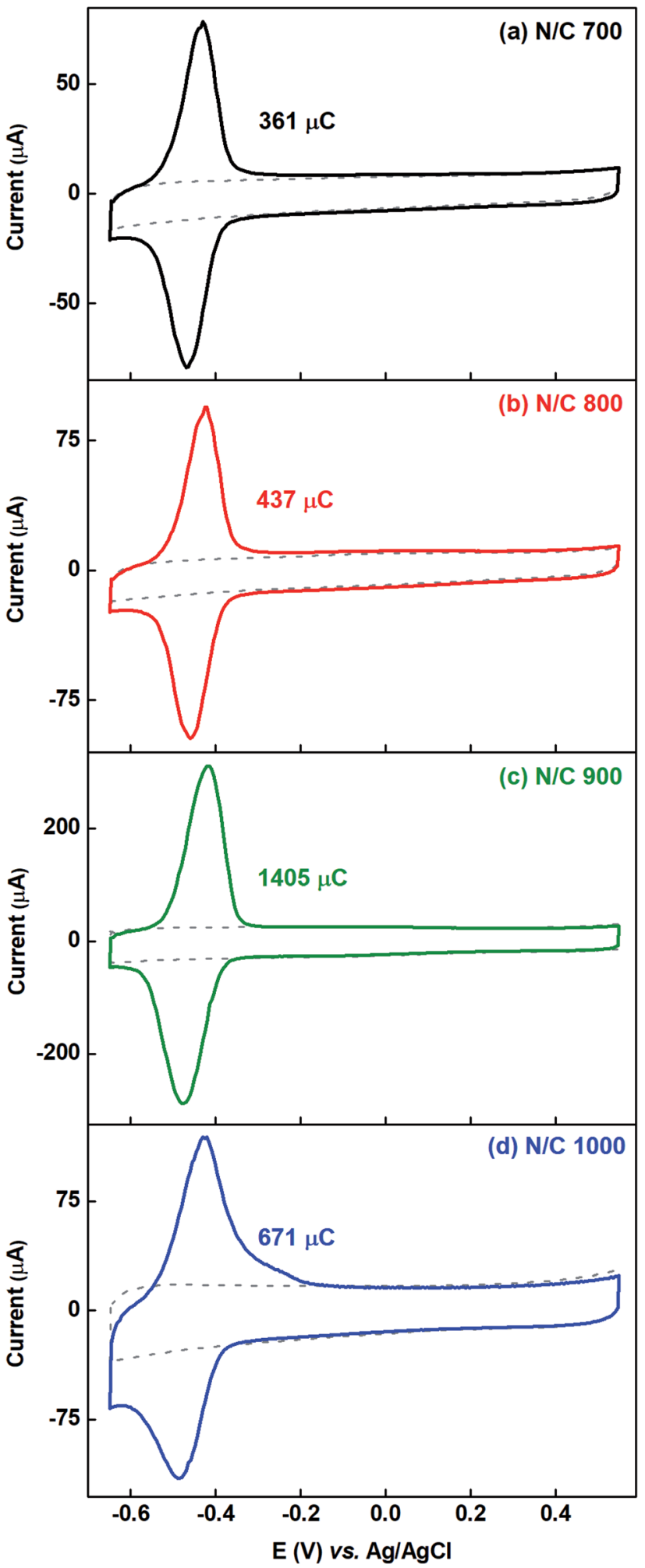

Fig. 6 Voltammograms of the AQS-adsorbed N/C 700 (a), N/C 800 (b), N/C 900 (c), and N/C 1000 (d) in argon-saturated 0.1 M PBS (pH 7) at $20 \mathrm{mV} \mathrm{s}^{-1}$ scan rate. The dotted gray line represents the background voltammogram of the respective catalyst.

estimate the charge due to adsorption of AQS, the background voltammogram is subtracted from the voltammogram of AQSadsorbed electrode. The charge due to oxidation of the adsorbed species is given in the respective figures and it is in the order N/C $900>\mathrm{N} / \mathrm{C} 1000>\mathrm{N} / \mathrm{C} 800>\mathrm{N} / \mathrm{C} 700$. This order is the same as that obtained in our previous work using catechol as a probe molecule. ${ }^{15}$ It was concluded that, N/C 900 has maximum active sites (mainly the nitrogen, oxygenated groups, edge sites etc.). Thus, the oxidation charge due to adsorbed-AQS on the N/C 900 is highest among all the nitrogen-doped catalysts.

The estimation method of active site density, ESA and the wetted surface area of the catalysts are discussed in the following section.

3.3.1.2 Estimation of the active site density, electrochemically active surface area (ESA) and wetted surface area. The adsorption charge measured from the voltammogram of AQS-adsorbed catalyst is used to estimate the active site density by the following eqn (1). ${ }^{15}$

$$
\begin{aligned}
& \mathrm{SD}_{\mathrm{M}}(\text { active sites per } \mathrm{g}) \\
& =\frac{\text { Integrated } \mathrm{CV} \text { area }(\mathrm{A} \cdot \mathrm{V}) \times N(\text { sites per mol })}{n \times \text { scan rate }\left(\mathrm{V} \mathrm{s}^{-1}\right) \times F\left(\mathrm{C} \mathrm{mol}^{-1}\right) \times m(\mathrm{~g})}
\end{aligned}
$$

In eqn (1), $\mathrm{SD}_{\mathrm{M}}$ is mass specific site density, $N$ is Avogadro number $\left(6.023 \times 10^{23}\right.$ sites per mol), $n$ is the number of electrons, $F$ is Faraday constant $\left(96485 \mathrm{C} \mathrm{mol}^{-1}\right.$ ), and $m$ is the mass of the catalyst $(26.6 \mu \mathrm{g})$ loaded on the GCE. The estimated site density per unit gram of catalyst is tabulated in Table 1 . The estimation method is based on the assumption that each active site on the catalyst surface adsorbs one AQS molecule. The number of electron transferred $(n)$ is taken as 2 for the calculation.

Eqn (2) is used to calculate the ESA of the catalysts and the normalizing factor is $611 \mu \mathrm{C} \mathrm{cm}_{\mathrm{c}}^{-2}$ (charge of unit surface area on graphite). It is obtained by multiplying the carbon atomic density $\left(3.82 \times 10^{15}\right.$ atom $\left._{\mathrm{c}} \mathrm{cm}_{\mathrm{c}}{ }^{-2}\right)$ with the electronic charge $\left(1.6 \times 10^{19} \mathrm{C} \mathrm{e}^{-1}\right) \cdot \cdot^{15,26}$

$$
\operatorname{ESA}\left(\mathrm{cm}_{\mathrm{c}}^{2} \mathrm{~g}^{-1}\right)=\frac{\mathrm{AQS}_{\mathrm{ads}} \text { charge }(\mathrm{C})}{2 \times m(\mathrm{~g}) \times 611 \mu \mathrm{C} \mathrm{cm}{ }_{\mathrm{c}}^{-2}}
$$

The estimated ESA of the catalysts is tabulated in Table 1.

The wetted surface area is estimated using the EIS as reported in the literature. ${ }^{15,27,28}$ In the Nyquist plot, the characteristics of an ideal capacitor is a vertical line. Whereas, in case of electrochemical capacitor, it generally starts with a $45^{\circ}$ line and approaches to a vertical line at low frequencies..$^{20,28}$ For an electrochemical capacitor, at low frequency, the capacitance generally becomes almost constant as usually observed in the capacitance $v$ s. frequency plot. ${ }^{29}$ At very low frequencies, where the imaginary part of the impedance dominates, the capacitance value extracted from the Nyquist plot follows the same trend as the capacitance measured from voltammetry or the current charge/discharge curves..$^{30,31}$ Thus, the double layer capacitance $\left(C_{\mathrm{dl}}\right)$ is measured using the eqn (3) given below.

$$
C_{\mathrm{dl}}=-\frac{1}{2 \pi f Z_{\mathrm{img}}}
$$


Table 1 Electrochemically active surface area (ESA) and wetted surface area of the catalysts

\begin{tabular}{llll}
\hline Catalyst & $\begin{array}{l}\text { Active site density } \\
\left(10^{20} \text { sites per } \mathrm{g}\right)\end{array}$ & $\begin{array}{l}\text { ESA (using adsorbed AQS) } \\
\left(10^{4} \mathrm{~cm}_{\mathrm{c}} \mathrm{g}^{-1}\right)\end{array}$ & $\begin{array}{l}A_{\text {wet }}(\text { using impedance) } \\
\left(10^{4} \mathrm{~cm}_{\mathrm{c}}{ }^{2} \mathrm{~g}^{-1}\right)\end{array}$ \\
\hline $\mathrm{C}$ & 0.32 & 0.83 & 18.19 \\
N/C 700 & 0.43 & 1.11 & 20.62 \\
N/C 800 & 0.52 & 1.34 & 21.83 \\
N/C 900 & 1.64 & 4.31 & 69.15 \\
N/C 1000 & 0.78 & 2.04 & 58.21 \\
$\mathrm{HNO}_{3}$ treated C & 0.33 & 0.86 & 105.76 \\
$\mathrm{HNO}_{3}$ treated N/C 900 & 0.64 & 1.66 & 188.01 \\
\end{tabular}

In eqn (3), the $Z_{\mathrm{img}}$ is the imaginary part of the impedance. The impedance data is recorded in the argon-saturated $0.1 \mathrm{M}$ PBS $(\mathrm{pH} 7)$ at $0.2 \mathrm{~V}$. The measured double layer capacitance $\left(C_{\mathrm{dl}}\right)$ of the catalysts is tabulated in Table S4. $\dagger$ The potential $0.2 \mathrm{~V}$ is chosen to record the impedance because no faradaic process is observed at this potential.

The wetted surface area of the sample is calculated using the measured double layer capacitance $\left(C_{\mathrm{dl}}\right)$ from EIS using the eqn $(4) .^{27,28}$

$$
C_{\mathrm{dl}}=\varepsilon_{0} \varepsilon_{\mathrm{r}} \frac{A_{\text {wet }}}{d}
$$

In eqn (4), $\varepsilon_{0}$ and $\varepsilon_{\mathrm{r}}$ are the absolute and relative dielectric permittivities of value $8.854 \times 10^{-12} \mathrm{~F} \mathrm{~m}^{-1}$ and 7 (the relative permittivity for inner Helmholtz layer), respectively. $A_{\text {wet }}$ is the wetted surface area, and $d$ is the double layer thickness $(\sim 0.2$ $\mathrm{nm})$.

3.3.1.3 Oxygen reduction reaction on the nitrogen-doped carbon in $0.1 \mathrm{M} \mathrm{KOH} \mathrm{media.} \mathrm{The} \mathrm{ORR} \mathrm{voltammograms} \mathrm{are}$ recorded in oxygen-saturated $0.1 \mathrm{M} \mathrm{KOH}$ medium for the nitrogen-doped carbon catalysts. Fig. S5† shows the ORR voltammograms of the nitrogen-doped carbon samples along with their Tafel plots as shown in our previous work. ${ }^{15}$ The kinetic current for the ORR is tabulated in Table 2. A comparison plot of active site density and ORR activity of different catalysts is provided in Fig. S6. $\dagger$

It is observed that for nitrogen-doped carbon catalysts, the estimated ESA (in Table 1), wetted surface area (in Table 1) and the ORR activity of the catalysts (in Table 2) are in the order N/C $900>$ N/C $1000>$ N/C $800>$ N/C 700 .

To further confirm the effectiveness of the proposed method, experiments were performed on the $\mathrm{HNO}_{3}$ treated catalyst and is discussed in the following section.

\subsubsection{Discussion on $\mathrm{HNO}_{3}$ treated catalysts}

3.3.2.1 Voltammograms of AQS-adsorbed $\mathrm{HNO}_{3}$ treated catalysts: differentiation between electrochemically active surface area (ESA) and wetted surface area. As representative catalysts, carbon and N/C 900 are treated with $\mathrm{HNO}_{3}$. The surface-nitrogen and oxygen content estimated from the XPS and the measured BET surface area are tabulated in Table S3. $\dagger$ After $\mathrm{HNO}_{3}$ treatment, the nitrogen content in the carbon sample does not change. But the oxygen content increases from $\sim 1$ to $\sim 11$ at $\%$. Whereas, for $\mathrm{N} / \mathrm{C} 900$, the oxygen content increases from $\sim 5.48$ to $\sim 16$ at $\%$. The BET surface area of the both catalysts decreases after acid treatment $\left(69 \% \mathrm{HNO}_{3}\right)$ and it is in the order of $\mathrm{N} / \mathrm{C} 900>\mathrm{C}>$ $\mathrm{HNO}_{3}$ treated N/C $900>\mathrm{HNO}_{3}$ treated C. Similar observation was reported by another group as well. ${ }^{23}$ It is found that the impurities from the carbon surface are removed on treatment with low concentration of $\mathrm{HNO}_{3}$ and the BET surface area increases. On treatment with $\mathrm{HNO}_{3}$ of higher concentration, the BET surface area decreases due to blocking of the pores and the surface is occupied with oxygenated groups. ${ }^{23}$

Fig. 7 shows the voltammograms of AQS-adsorbed carbon and N/C 900 along with that of the $\mathrm{HNO}_{3}$ treated samples. The estimated charge due to adsorbed-AQS is given in the respective figures. After $\mathrm{HNO}_{3}$ treatment of the carbon catalyst, almost no change is observed in the charge due to adsorption of AQS, whereas, it decreases after $\mathrm{HNO}_{3}$ treatment for N/C 900. The measured active site density changes from $1.64 \times 10^{20}$ to $0.64 \times$ $10^{20}$ sites per $\mathrm{g}$ for N/C 900 catalysts as given in the Table 1 . One of the possible reasons may be the decrement in the BET surface area of the N/C 900 from $\sim 460 \mathrm{~m}^{2} \mathrm{~g}^{-1}$ to $\sim 125 \mathrm{~m}^{2} \mathrm{~g}^{-1}$, possibly due to blocking of the pores. Whereas, after $\mathrm{HNO}_{3}$ treatment, the wetted surface area of the carbon and the N/C 900 catalyst increases significantly in comparison to their non-treated sample. The estimated wetted surface area is given in Table 1.

Table 2 Kinetic current density and the turnover frequency (TOF) of the catalysts towards the ORR

\begin{tabular}{|c|c|c|c|}
\hline Catalyst & $\begin{array}{l}\text { ORR current density } I_{\mathrm{k}}\left(\mathrm{mA} \mathrm{cm}_{\mathrm{g}}{ }^{-2}\right) \\
\text { at }-0.25 \mathrm{~V} v s . \mathrm{Ag} / \mathrm{AgCl}\end{array}$ & $\begin{array}{l}\text { ToF }(\text { at }-0.25 \mathrm{~V} \\
\text { vs. } \mathrm{Ag} / \mathrm{AgCl})\left(\mathrm{e}^{-} \text {per site per } \mathrm{s}\right)\end{array}$ & $\begin{array}{l}\text { ToF (at } 0.8 \mathrm{~V} \\
\text { vs. RHE) }\left(\mathrm{e}^{-} \text {per site per } \mathrm{s}\right)\end{array}$ \\
\hline $\mathrm{C}$ & 0.260 & 0.36 & 0.019 \\
\hline N/C 700 & 0.593 & 0.63 & 0.033 \\
\hline N/C 800 & 1.044 & 0.92 & 0.072 \\
\hline N/C 900 & 6.039 & 1.69 & 0.248 \\
\hline N/C 1000 & 2.246 & 1.32 & 0.148 \\
\hline $\mathrm{HNO}_{3}$ treated $\mathrm{C}$ & 0.371 & 0.53 & 0.020 \\
\hline $\mathrm{HNO}_{3}$ treated N/C 900 & 1.302 & 0.37 & 0.021 \\
\hline
\end{tabular}




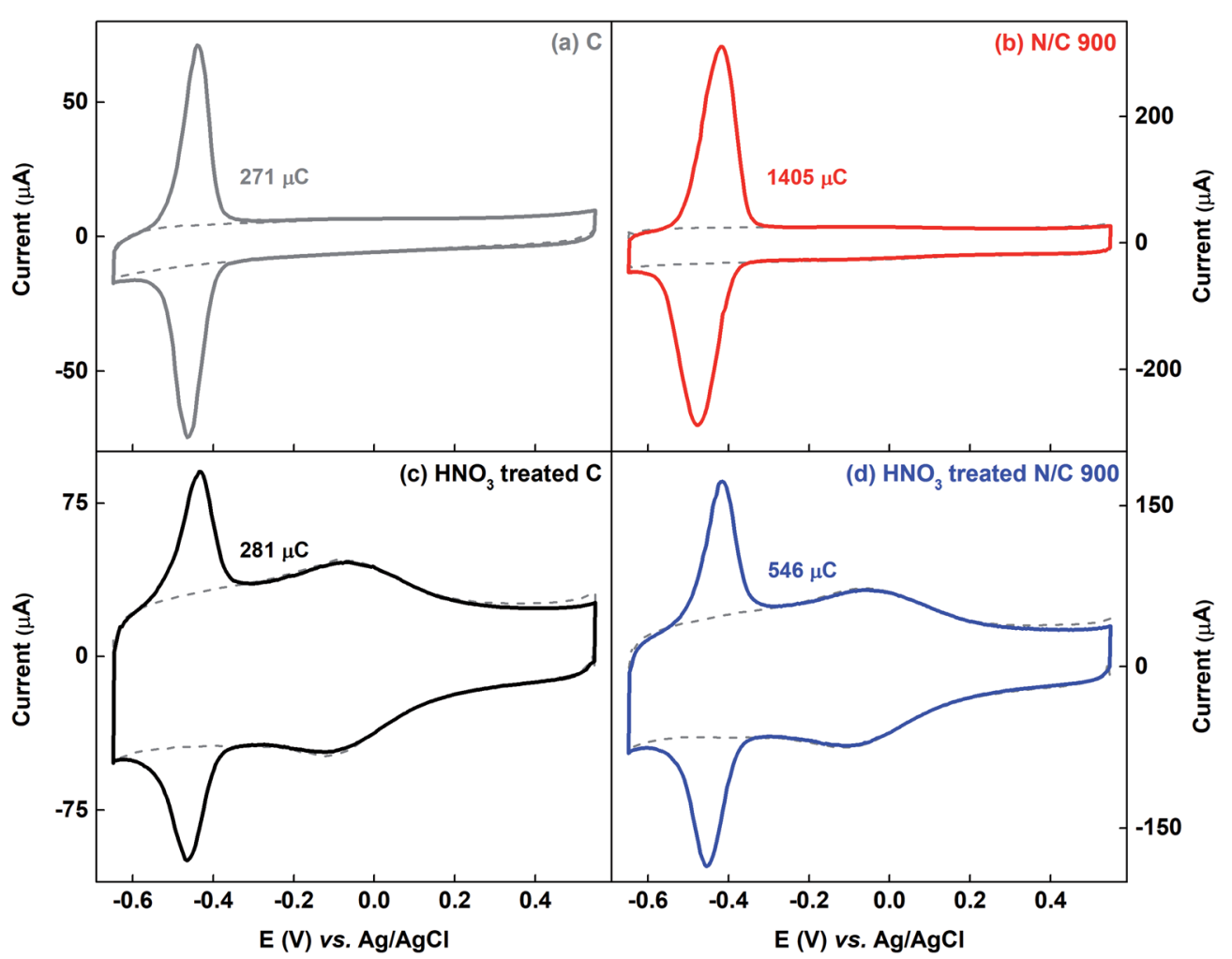

Fig. 7 Voltammograms of the AQS-adsorbed C (a), N/C 900 (b), $\mathrm{HNO}_{3}$ treated C (c), and $\mathrm{HNO}_{3}$ treated N/C 900 (d) in argon-saturated $0.1 \mathrm{M}$ PBS $(\mathrm{pH} 7)$ at $20 \mathrm{mV} \mathrm{s}^{-1}$ scan rate. The dotted gray line represents the background voltammogram of the respective catalyst.

Thus, while comparing, it is observed that the wetted surface area of the catalysts increases after $\mathrm{HNO}_{3}$ treatment, though, the ESA does not follow the same trend.

In the next section, a comparison of the activity towards the ORR is shown for carbon and N/C 900 with their respective $\mathrm{HNO}_{3}$ treated catalysts.

3.3.2.2 Comparison of oxygen reduction reaction on the $\mathrm{HNO}_{3}$ treated and untreated catalyst in $0.1 \mathrm{M} \mathrm{KOH} \mathrm{media.} \mathrm{The} \mathrm{ORR}$ voltammograms are recorded in oxygen-saturated $0.1 \mathrm{M} \mathrm{KOH}$ medium. Fig. 8 shows the comparison plot of the ORR voltammograms for carbon and N/C 900 along with their $\mathrm{HNO}_{3}$ treated catalysts. The Tafel plots are shown in the inset of the Fig. 8. The activity of catalysts is tabulated as kinetic current in Table 2.

After $\mathrm{HNO}_{3}$ treatment, the activity of the carbon is almost constant and the activity of N/C 900 decreases; the trend is N/C $900>\mathrm{HNO}_{3}$ treated N/C $900>\mathrm{HNO}_{3}$ treated $\mathrm{C} \approx \mathrm{C}$. This order is the same as the ESA of the catalysts estimated using the AQSadsorption technique; the wetted surface area does not follow the same order.

Thus, it can be concluded that the ESA of the catalyst is different from that of wetted surface area of the catalyst; using AQS adsorption method active site density can be estimated.

3.3.3 Estimation of turnover frequency (ToF). The turnover frequency (ToF) of the catalysts in $\mathrm{e}^{-}$per site per $\mathrm{s}$ towards ORR is calculated using the eqn (5).

$$
I_{\mathrm{M}(\text { fixed E) }}=\mathrm{SD}_{\mathrm{M}} \times \mathrm{ToF}_{(\text {fixed E) }} \times e
$$

In eqn (5), $I_{\mathrm{M}}$ is the mass activity $\left(\mathrm{A} \mathrm{g}^{-1}\right)$, the kinetic current normalized by the mass of the loaded catalyst on the glassy carbon electrode, $\mathrm{SD}_{\mathrm{M}}$ is the site density (sites per $\mathrm{g}$ ), and $e$ is the electronic charge $\left(1.6 \times 10^{-19} \mathrm{C}\right)$.

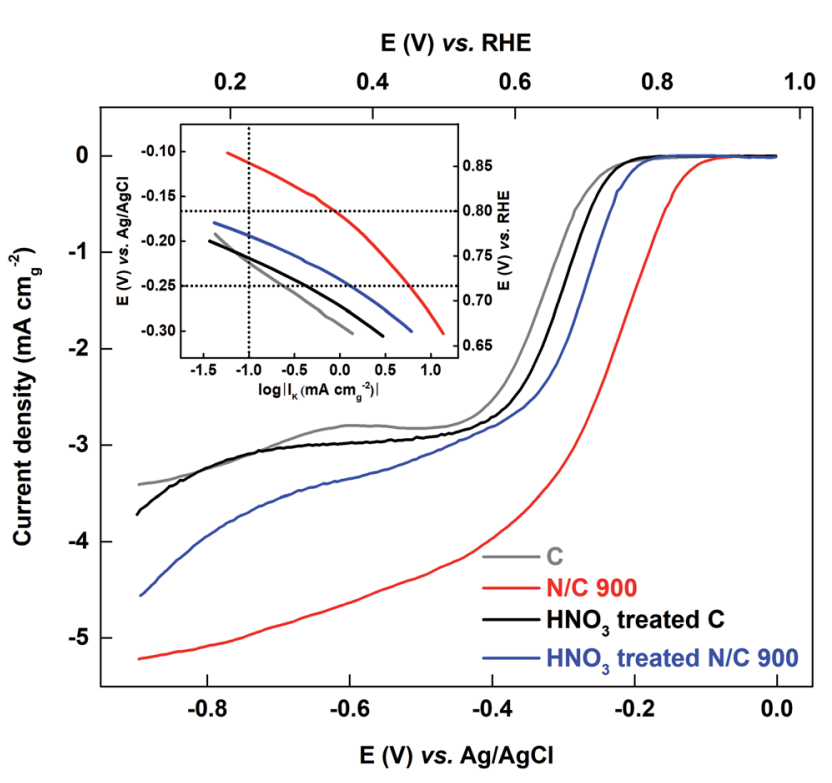

Fig. 8 ORR voltammograms of $\mathrm{C}$ and N/C 900 with their respective $\mathrm{HNO}_{3}$ treated catalysts recorded in oxygen-saturated $0.1 \mathrm{M} \mathrm{KOH}$ solution ( $\mathrm{pH}$ 13) at a scan rate of $20 \mathrm{mV} \mathrm{s}^{-1}$ at $1600 \mathrm{rpm}$. Inset shows the Tafel plot of the respective catalysts. 
The ToF value at $-0.25 \mathrm{~V} v s$. $\mathrm{Ag} / \mathrm{AgCl}$ is calculated using the eqn (5) based on the site density measured using the adsorbedAQS and is given in the Table 2. Except N/C 900 and N/C 1000, all the other catalysts show low kinetic current density (below $0.1 \mathrm{~mA} \mathrm{~cm}_{\mathrm{g}}{ }^{-2}$ ) at $0.8 \mathrm{~V} v$ s. RHE. Thus, the ToF values needs to be extrapolated for other catalysts. The obtained ToF value at $-0.25 \mathrm{~V} v s$. $\mathrm{Ag} / \mathrm{AgCl}$ is extrapolated to $0.8 \mathrm{~V} v s$. RHE using the eqn (6) given below. ${ }^{10,32}$

$$
\begin{aligned}
\operatorname{ToF}(0.8 \mathrm{~V} v s . \mathrm{RHE})= & \operatorname{ToF}(0.5 \mathrm{~V} v s . \mathrm{Ag} / \mathrm{AgCl}) \\
& \times \exp \left[\frac { - \operatorname { l n } 1 0 } { \text { Tafel } } \left(E_{0.8 \text { vs. } \mathrm{RHE}}\right.\right. \\
& \left.\left.-E_{0.5 \text { vs. } \mathrm{Ag} / \mathrm{AgCl}}\right)\right]
\end{aligned}
$$

The estimated ToF values towards ORR of the catalysts at $0.8 \mathrm{~V} v s$. RHE are tabulated in Table 2. It is observed that for the carbon and nitrogen-doped catalysts the ToF value is in the range of $0.019-0.248 \mathrm{e}^{-}$per site per $\mathrm{s}$, which is slightly lower than the previously reported value using the catecholadsorption method (0.059-0.568 $\mathrm{e}^{-}$per site per s). ${ }^{15}$ The estimated ToF values for carbon and nitrogen-doped carbon catalysts are almost one to two orders of magnitude lower than that reported with $\mathrm{Fe}-\mathrm{N} / \mathrm{C}$ catalysts $\left(\sim 1.5\right.$ or $1.6 \mathrm{e}^{-}$per site per $\left.\mathrm{s}\right)$ using nitrite adsorption and combined Mössbauer and CO adsorption methods. ${ }^{\mathbf{8 1 2}}$ Guo et al. reported the ToF values for the nitrogen-doped HOPG and nitrogen-doped graphene at $0.5 \mathrm{~V} v s$. RHE and it is $0.07-0.14 .^{26}$ Instead of ESA, the active BET surface area of the catalysts was taken for normalization. The active BET surface area was calculated by multiplying the BET surface area with possible active nitrogen content in the catalysts estimated using the XPS.

\section{Conclusions}

Voltammograms of AQS-adsorbed carbon and nitrogen-doped carbon surfaces are recorded in the argon-saturated 0.1 M PBS $(\mathrm{pH} 7)$ solution. The charge due to adsorbed-AQS represents the active site density, viz., the ESA and it is in the order of N/C $900>$ N/C $1000>$ N/C $800>$ N/C $700>C$. A similar trend is observed for the activity towards oxygen reduction reaction recorded in alkaline media. Besides, the turnover frequency (ToF) of the catalysts towards oxygen reduction is calculated using the estimated charge. The ToF is in line with the data reported using other methods in the literature for NPMCs. Moreover, carbon and N/C 900 catalysts are functionalized by the $\mathrm{HNO}_{3}$ treatment. It is observed that, after functionalization, though the double layer capacitance or the wetted surface area of the catalyst increases, the ESA for the carbon is almost constant and for the N/C 900 catalyst it decreases; the ORR activity of the catalysts follows the same trend. It suggests that the wetted surface area and the ESA do not follow the same trend.

In comparison to catechol adsorption method, AQS provides single, reproducible, and saturated voltammetric peak for adsorption over a broader concentration range and potential window. No unwanted side reaction is observed, which makes the process more reliable. Furthermore, the surface-adsorbed AQS can be removed easily by the proposed electrochemical cleaning method; the clean surface can be used for further electrochemical characterization.

\section{Conflicts of interest}

There are no conflicts of interest to declare.

\section{Acknowledgements}

The authors would like to acknowledge National Centre for Photovoltaic Research and Education (NCPRE, P16MNRE002) Phase-II and India-UK centre for Education and Research in Clean Energy (IUCERCE, 16DST050) for financial support to carry out the work. The Department of Metallurgical Engineering and Materials Science (MEMS) and the Department of Physics, both at IIT Bombay, are acknowledged for allowing us to use the facility to measure the BET surface area and to record the XPS spectra, respectively.

\section{References}

1 H. A. Gasteiger, S. S. Kocha, B. Sompalli and F. T. Wagner, Appl. Catal., B, 2005, 56, 9-35.

2 H. A. Gasteiger and N. M. Markovic, Science, 2009, 324, 4849.

3 F. J. Vidal-Iglesias, R. M. Arán-Ais, J. Solla-Gullón, E. Herrero and J. M. Feliu, ACS Catal., 2012, 2, 901-910.

4 J. Solla-Gullón, P. Rodríguez, E. Herrero, A. Aldaz and J. M. Feliu, Phys. Chem. Chem. Phys., 2008, 10, 1359-1373.

5 R. Devivaraprasad, R. Ramesh, N. Naresh, T. Kar, R. K. Singh and M. Neergat, Langmuir, 2014, 30, 8995-9006.

6 R. Rahul, R. K. Singh and M. Neergat, J. Electroanal. Chem., 2014, 712, 223-229.

7 A. Cuesta, A. Couto, A. Rincón, M. C. Pérez, A. López-Cudero and C. Gutiérrez, J. Electroanal. Chem., 2006, 586, 184-195.

8 D. Malko, A. Kucernak and T. Lopes, Nat. Commun., 2016, 7, 13285-13292.

9 S. Gupta, C. Fierro and E. Yeager, J. Electroanal. Chem., 1991, 306, 239-250.

10 F. Jaouen and J. P. Dodelet, Electrochim. Acta, 2007, 52, 59755984.

11 U. I. Kramm, J. Herranz, N. Larouche, T. M. Arruda, M. Lefèvre, F. Jaouen, P. Bogdanof, S. Fiechter, I. A. Wurmbach, S. Mukerjee and J. P. Dodelet, Phys. Chem. Chem. Phys., 2012, 14, 11673-11688.

12 A. Zitolo, V. Goellner, V. Armel, M. T. Sougrati, T. Mineva, L. Stievano, E. Fonda and F. Jaouen, Nat. Mater., 2015, 14, 937-942.

13 J. L. Kneebone, S. L. Daifuku, J. A. Kehl, G. Wu, H. T. Chung, M. Y. Hu, E. E. Alp, K. L. More, P. Zelenay, E. F. Holby and M. L. Neidig, J. Phys. Chem. C, 2017, 121, 16283-16290.

14 N. R. Sahraie, U. I. Kramm, J. Steinberg, Y. Zhang, A. Thomas, T. Reier, J. P. Paraknowitsch and P. Strasser, Nat. Commun., 2015, 6, 8618-8627. 
15 A. Chakraborty, R. Devivaraprasad, B. Bera and M. Neergat, Phys. Chem. Chem. Phys., 2017, 19, 25414-25422.

16 A. Kanazawa, T. Daisaku, T. Okajima, S. Uchiyama, S. Kawauchi and T. Ohsaka, Langmuir, 2014, 30, 5297-5305.

17 G. G. Wildgoose, A. T. Masheter, A. Crossley, J. H. Jones and R. G. Compton, Int. J. Electrochem. Sci., 2007, 2, 809-819.

18 M. D. Ryan, A. Yueh and W. Y. Chen, J. Electrochem. Soc., 1980, 127, 1489-1495.

19 C. R. Dawson and W. B. Tarpley, Ann. N. Y. Acad. Sci., 1963, 100, 937-950.

20 B. Bera, T. Kar, A. Chakraborty and M. Neergat, J. Electroanal. Chem., 2017, 805, 184-192.

21 B. Bera, A. Chakraborty, T. Kar, P. Leuaa and M. Neergat, J. Phys. Chem. C, 2017, 121, 20850-20856.

22 M. Carmo, M. Linardi and J. G. R. Poco, Appl. Catal., A, 2009, 355, 132-138.

23 J. Li, L. Ma, X. Li, C. Lu and H. Liu, Ind. Eng. Chem. Res., 2005, 44, 5478-5482.

24 T. Kar, R. Devivaraprasad, B. Bera, R. Ramesh and M. Neergat, Electrochim. Acta, 2016, 191, 81-89.
25 M. Quan, D. Sanchez, M. F. Wasylkiw and D. K. Smith, J. Am. Chem. Soc., 2007, 129, 12847-12856.

26 D. Guo, R. Shibuya, C. Akiba, S. Saji, T. Kondo and J. Nakamura, Science, 2016, 351, 361-365.

27 J. Friedl, C. M. Bauer, A. Rinaldi and U. Stimming, Carbon, 2013, 63, 228-239.

28 J. Friedl and U. Stimming, Electrochim. Acta, 2017, 227, 235245.

29 R. Kötz and M. Carlen, Electrochim. Acta, 2000, 45, 24832498.

30 C. Cougnon, E. Lebègue and G. Pognon, J. Power Sources, 2015, 274, 551-559.

31 P. L. Taberna, P. Simon and J. F. Fauvarque, J. Electrochem. Soc., 2003, 150, A292-A300.

32 F. Jaouen, J. Herranz, M. Lefèvre, J. P. Dodelet, U. I. Kramm, I. Herrmann, P. Bogdanoff, J. Maruyama, T. Nagaoka, A. Garsuch, J. R. Dahn, T. Olson, S. Pylypenko, P. Atanassov and E. A. Ustinov, ACS Appl. Mater. Interfaces, 2009, 1, 1623-1639. 